\title{
On The Histochemical Reactions for Uridine Diphosphoglucose Glycogen Transferase and Phosphorylase in The Normal Skeletal Muscles
}

\author{
Tadao TAKeuchi and Yasuya Hiratr \\ Department of Pathology, Kumamoto University School of Medicine, Kumamoto.
}

Takeuchi ${ }^{1-6)}$ stated that there are two major pathways utilizing the amylophosphorylase $\rightarrow$ branching enzyme (amylo-1,4 $\rightarrow 1,6$-transglucosidase) system and the pyrophosphorylase $\rightarrow$ UDPG-glycogen transferase system for the synthesis of glycogen from glucose-1-phosphate in muscle fibers as well as in certain tissue cells from a histochemical point of view. Villar-Palasi and Larner ${ }^{7}$ maintained biochemically that the UDPG-glycogen transferase completes the final step in conversion of glucose-1-phoshate to glycogen and that phosphorylase degrades glycogen to supply muscle with hexose phosphate as an energy source. Hess and Pearse ${ }^{9)}$ suggested that the histochemical study on the distribution pattern of these enzymes in resting skeletal muscle of the rat revealed a reciprocal relationship between phosphorylase and UDPG-glycogen transferase in individual fibers. The former was operative in large white fibers and the latter in small red fibers. Thus, the activity for these enzymes in individual fibers of the normal skeletal muscle was observed in detail from our histochemical point of view in this paper.

\section{Methods}

Muscle tissues, excised from rat and rabbit bled under anesthesia and from human being at the surgical operation, were quenched on dry ice and were cut at $10 \mu$ on a cold microtome in cryostat at $-20^{\circ} \mathrm{C}$ and mounted on slides. The activity of phosphorylase, branching enzyme and UDPG-glycogen transferase was demonstrated according to Takeuchi ${ }^{1,2,5,7)}$. The activity of phosphorylase $a$ and phosphorylase $b$ activated by phosphorylase $b$-kinase also was demonstrated by use of Takeuchi and Hirata's prcocedure ${ }^{10}$.

\section{Results}

A striking difference in each enzyme activity was noted in muscle fibers of varying size (Fig. 1-6). The phosphorylase activity demonstrated in the Takeuchi original medium was strongly active in large fibers of the human, rabbit and rat under the normal resting condition. The reaction for phosphorylase in small fibers occurred usually lower, but occasionally appeared to occur intensely in certain skeletal muscles. The phosphorylase $a$ demonstrated by incubating in the Takeuchi original medium containing alcohol with adenosine- $5^{\prime}$-phosphate (AMP) as well as without AMP appeared to be present 
intensely in large fibers (Fig. 1, 4). The total phosphorylases activated by addition of adenosine triphosphate (ATP), Mg-ions and glucagon to the Takeuchi mixture containing AMP and alcohol were strongly demonstrable in small fibers as well as in large fibers (Fig. 2). It was accordingly found that phosphorylase $b$ rather than $a$ predominated in small fibers. On the other hand, high activity of branching enzyme was obtained in small fibers (Fig. $5,6)$. In other word, polysaccharide stained reddish violet with iodine was histochemically synthesized from glucose-1-phosphate in small fibers, while polysaccharide stained deep blue with iodine was obtained in large fibers from it.

The activity of UDPG-glycogen tranferase was strongly demonstrable in small fibers rather than in large fibers, with the exception of some fibers (Fig. 3, 7). There was, however, not so much difference between small and large fibers in UDPG-glycogen transferase as in phosphorylase (Fig. 7). Polysaccharide formed by UDPG-glycogen transferase always stained red brown with iodine.

Optimal $\mathrm{pH}$ for each histochemical reaction was somewhat different. Polysaccharide formation from the substrate was made most suitably at pH 5.7 by phosphorylase, at $\mathrm{pH} 6.0$ to 6.4 by branching enzyme and at $\mathrm{pH} 7.2$ to 7.6 by UDPG-glycogen transferase. The UDPG-glycogen transferase reaction was activated by substituting glucose-6-phosphate in the medium, while the phosphorylase reaction was inhibited by it. The branching enzyme was intensely inhibited by alcohol and $\mathrm{HgC}_{2}$. The total phosphorylase reaction was activated by ATP, $\mathrm{Mg}^{++}$, and glucagon and $\mathrm{KIO}_{3}$.

\section{Discussion}

It was histochemically proved that there are two enzymatic pathways for glycogen synthesis in muscle fibers (Fig. 8). In this case, although polysaccharide stained blue with iodine can be histochemically synthesized in muscle fibers removed from animal, such polysaccharide is never found in muscle fibers under the physiological condition without incubation in the substrate medium. The native glycogen in muscle stains always red brown with iodine, even if it remains in tissue after removing. In other words, the iodine-blue polysaccharide formation occurs in muscle fibers in vitro, but it does not occur in vivo. It was already found that the action of UDPG-glycogen transferase is a formation of 1,4-linkage to plysaccharide, and that the branching enzyme makes a branch formation of $1,4 \rightarrow 1,6$-linkage to polysaccharide. These facts assume the existence of a cyclic pathway for glycogen synthesis and breakdown in vivo, and the histochemical reaction for phosphorylase seems to represent only the activity, apart from the synthetic reaction (Fig. 9).

In muscle fibers under the physiological condition, it seems to be suggested that the synthesis of glycogen occurs always in every fibers and synthesized glycogen is broken down and utilized stronger in large fibers than in small fibers as an energy source. Histochemical reduction of UDPG-glycogen transferase in large fibers may reveal the fact as Pearse's suggestion that polysac- 


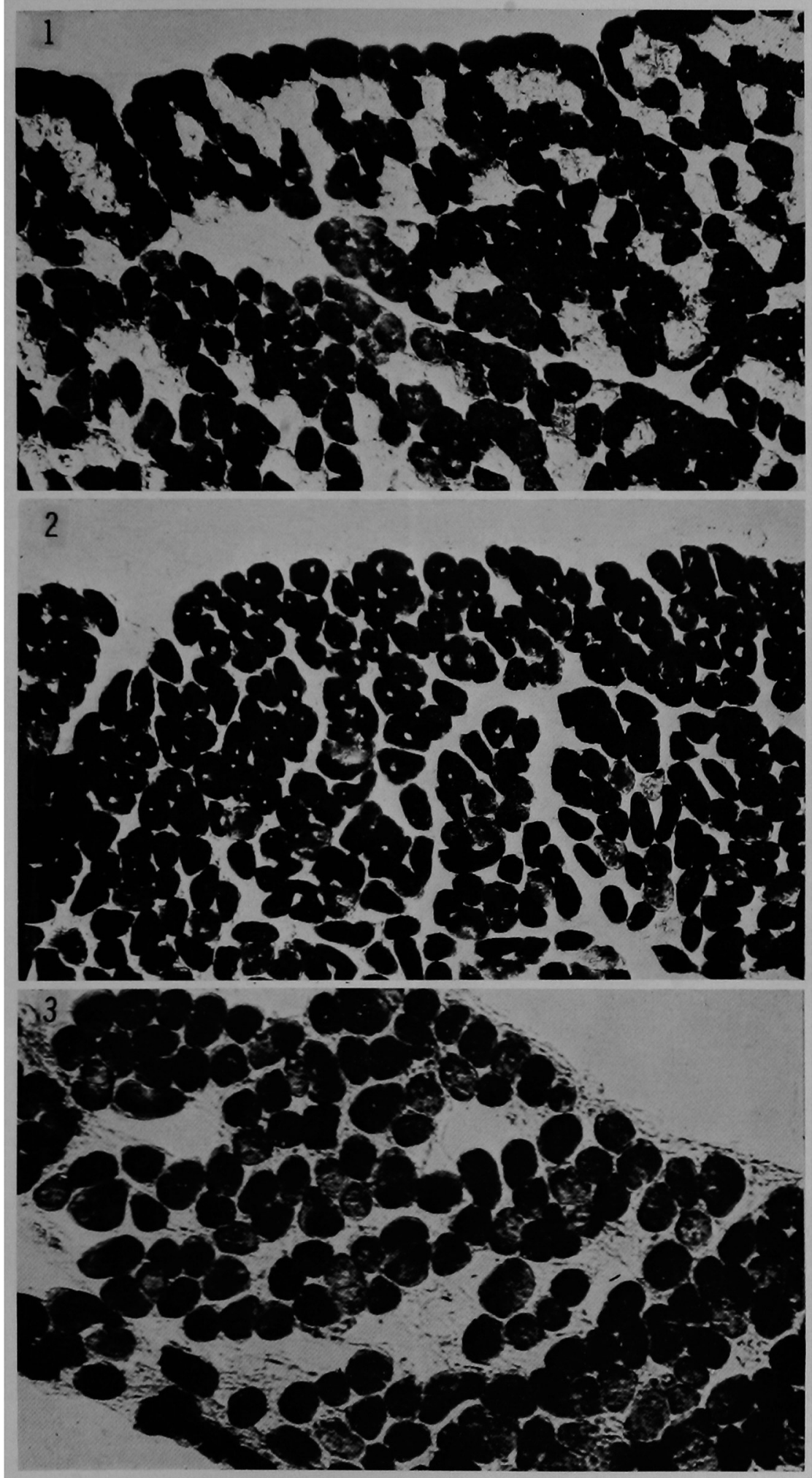




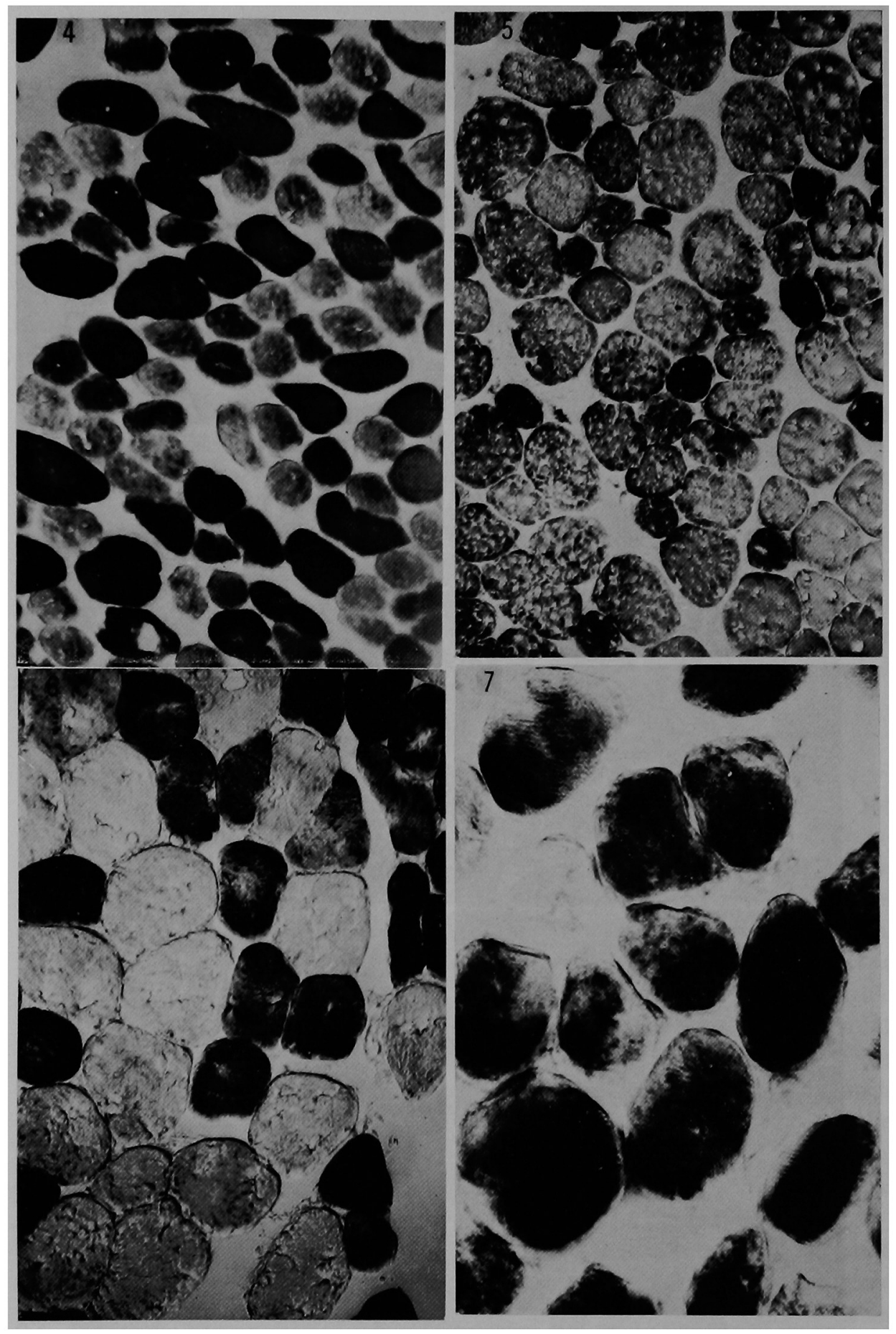




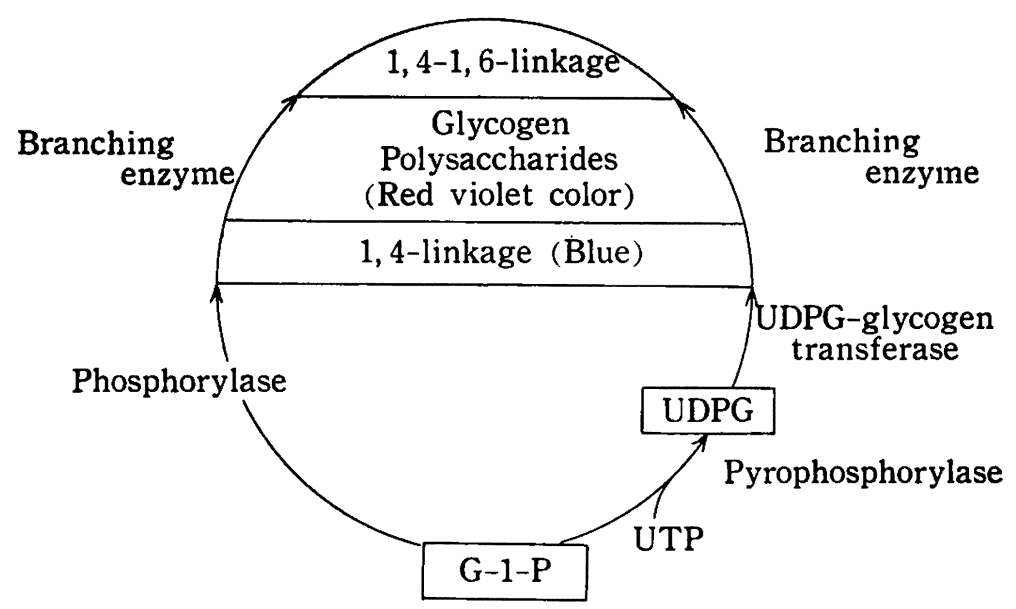

Fig. 8. Two synthetic pathways in vitro, histochemistry

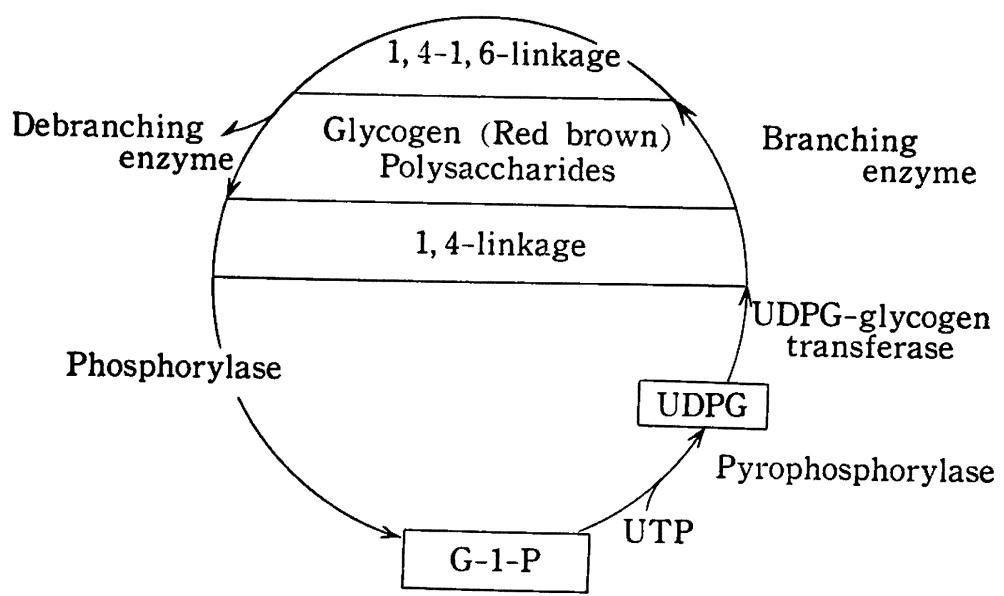

Fig. 9. A cyclic pathway in vivo

Branching enzyme; Amylo-1,4 $\rightarrow$ 1,6-transglucosidase

Debranching enyme; Amylo-1,6-glucosidase, G-1-P; Glucose-1-phosphate UTP ; Uridine triphosphate, UDPG; Uridine diphosphate glucose

Fig. 1 Phosphorylase $a$ is demonstrated predominantly in large pyramidal fibers of the rat diaphragma muscle by incubating in the Takeuchi original substrate mixture.

Fig. 2 Total phosphorylases (phosphorylase $a$ and phosphorylase $b$ activated by phosphorylase $b$-kinase together) are demonstrated in every muscle fibers of the same serial section.

Fig. 3 UDPG-glycogen transferase is demonstrated in every fibers, but demonstrable rather predominantly in small fibers of the same serial section.

Fig. 4 The phosphorylase reaction of the rat femoral muscle is detected by incubating in the Takeuchi original mixture. It is rather strongly demonstrable in large fibers than in small fibers.

Fig. 5 Branching enzyme is more intensely demonstrable in small fibers than in large fibers of the rabbit femoral muscle.

Fig. 6 Branching enzyme is intensely detected in small fibers of the human gluteal muscle.

Fig. 7 UDPG-glycogen transferase is demonstrated in individual fibers of the human abdominal muscle. The strength of the activity is not distinguishable between in the small fibers and in the large. 
charide synthesized histochemically from UDPG may be broken down rapidly by the phosphorylase present in excess. Besides, as the energy metabolism in small fibers does not need so much glycogen as in large fibers, the polysaccharide formed by UDPG-glycogen transferase seems to be deposited predominantly in the former. These suggestions may support the fact that large fibers of musle are supplied with the pyramidal innervtion and have a kinetic function needing high energy. It seems, moreover, to be related to the extrapyramidal and tonic function in small fibers that the activity of phosphorylase $a$ demonstrated with AMP is lower, the phosphorylase $b$ activated with phosphorylase $b$-kinase predominates, and the total phosphorylase is strongly concentrated in these small fibers. Glycogen synthesis and breakdown in muscle fibers seems to be also influenced by the intracellular biochemical conditions such as $\mathrm{pH}$, co-enzymes, activators and inhibitors (Takeuchi ${ }^{1,7)}$ ).

\section{Summary}

Both UDPG-glycogen transferase and total phosphorylase are strongly concentrated in small fibers as well as in large fibers of skeletal muscles. Phosphorylase $a$ predominates in large fibers and phosphorylase $b$ predominates in small fibers under the normal resting condition. The branching enzyme tends to reveal same distribution pattern as UDPG-glycogen transferase. Glycogen metabolism which is able to show histochemically two synthetic pathways in vitro seems to proceed physiologically through a cyclic pathway in vivo. It is considered that the large fibers contain rather intensely active phosphorylase in order to pyramidal kinetic function and the small fibers contain predominantly inactive phosphorylase activated by phosphorylase $b$-kinase for extrapyamidal tonic function. The activity of these enzymes must be changeable under varied functional conditions in individual fibers.

\section{References}

1) Takeuchi, T. Ann. Histochim. $7: 61-80,1962$.

2) Takeuchi, T. J. Histochem. $4: 84$, 1956. 3) Takeuchi, T. \& Kuriaki, H. J. Histochem. Cytochem. $3: 153-160,1955.4$ 4) Takeuchi, T., Higashi, K. \& Watanuki, S. J. Histochem. Cytochem. 3 : 485-491, 1955. 5) Takeuchi, T. J. Histochem. Cytochem. $6: 208-216,1958$. 6) Takeuchi, T. \& Glenner, G. G. J. Histochem. Cytochem. $8: 277-230,1960 . \quad 7)$ Takeuchi, T. \& Glenner, G. G. J. Histochem. Cytochem. $9: 304-316,1961 . \quad 8)$ Villar-Palasi, C. \& Larner, J. Arch. Biochem. Biophys. 86 : 270-273, 1950 . 9) Hess, R. \& Pearse, A.G.E. Proc. Sco. Exper. Biol. Med. $107: 569-571,1961 . \quad 10)$ Takeuchi, T. \& Hirata, Y. Kumamoto Med. J. $14: 188,1961$.

\section{Discussion}

Dr. Ogata :

1) In mammalia, bird, fish, reptilia and amphibia, the striated muscles are divided in three types through oxidative enzyme reactions (succinoxidase, diaphorases, and some dehydrogenases); the small red fiber with high enzyme activities, large white fiber with low activities and medium fiber with medium activities. What do you think of the medium fiber?

2) In warm-blooded animals, phosphorylase reaction (by Takeuchi) was strong in the white muscle fibers and weak in the red fibers, and on the other hand, the reverse 
results were obtained in cold-blooded animals.

Dr. Takeuchi :

1) The red and white fibers really show different activities of UDPG-glycogen transferase and phosphorylase, but the existence of the "medium fiber" should be further studied because intensity of enzyme reactions may be effected and regulated by various conditions.

2) I can not agree with you about the second problems. For example, the red fiber of human striated mucles may occasionally show high activity of phosphorylase and the white fiber of frog also does.

\title{
Studies on the Thiolacetic acid method for Histochemical Demonstration of Cholinesterase and Its Distribution in Muscles
}

\author{
Takao Muro, Hitoshi Tanabe, Satoshi Ueda, Masanori, Tomonaga \\ and Masanori Uono \\ From the third Department of Internal Medicine (Chief: Prof. S. Okinaka), \\ Faculty of Medicine, University of Tokyo
}

\begin{abstract}
Introduction
Since Gomori's histochemical method (1948) ${ }^{1)}$ for cholinestestese (ChE) using high fatty acid choline ester as substrate had been reported, Koelle's thicholine method (19492), 19503), 1951'), Ravin, Zsou \& Seligman's azo-dye method (1951) ${ }^{5)}$, Barrnett \& Seligman's (1951) and Holt \& Withers' (1952) ${ }^{7)}$ indoxyl methods were successively reported. Thereafter, Crevier \& Bélanger $(1955)^{8)}$ reported a new histochemical method for $\mathrm{ChE}$ using thiolacetic acid as substrate. The principle of this method was based on Wilson's researches ${ }^{9}$ in which thiolacetic acid was hydrolysed by $\mathrm{AChE}$ at an acid $\mathrm{pH}$ with the formation of acetic acid and hydrogen sulfide. Practically it consisted of an incubation with thiolacetic acid and lead nitrate, and liberated hydrogen sulfide at $\mathrm{ChE}$ active sites was precipitated as dark brown lead sulfide. Later on, this method was modified by Csillik \& Sávay ${ }^{10,11)}$ and recently by Wachstein, Meisel \& Falcon ${ }^{12)}$. Csillik \& Sávay chiefly investigated about motor end-plate by their modified method and protargol impregnation as counterstaining.

In this report, we studied the distribution of $\mathrm{ChE}$ activity in several organs or tissues by the thiolacetic acid method as comparing with the thiocholine method. Further, by that method, the distribution of $\mathrm{ChE}$ in muscle of several species was investigated.
\end{abstract}

